\title{
THE AAO 2DF QSO REDSHIFT SURVEY
}

\author{
B.J.BOYLE \\ Anglo-Australian Observatory \\ PO Box 296, Epping, NSW 2121, Australia \\ R.J.SMITH \\ Institute of Astronomy \\ Madingley Road, Cambridge CB3 OHA, UK \\ T.SHANKS AND S.M.CROOM \\ Department of Physics, University of Durham \\ South Road, Durham DH1 3LE, UK \\ AND \\ L.MILLER \\ Department of Physics, University of Oxford \\ Keble Road, Oxford OX1 3RH, UK
}

\section{Introduction}

The study of large-scale structure through QSO clustering provides a potentially powerful route to determining the fundamental cosmological parameters of the Universe (see Croom \& Shanks 1996). Unfortunately, previous QSO clustering studies have been limited by the relatively small sizes of homogeneous QSO catalogues that have been available. Although approximately 10,000 QSOs are now known (Veron-Cetty \& Veron 1997), the largest catalogues suitable for clustering studies contain only 500-1000 QSOs (Boyle et al. 1990, Crampton et al. 1990, Hewett et al. 1994). Even combining all such suitable catalogues, the total number of QSOs which can be used for clustering studies is still only about 2000 .

From these catalogues, significant $(5 \sigma)$ QSO clustering has been detected at small scales $r<10 \mathrm{~h}^{-1} \mathrm{Mpc}$ (Shanks \& Boyle 1994), with a correlation length $r_{0}=6 \mathrm{~h}^{-1} \mathrm{Mpc}$. However, these results are based on samples of QSOs covering a wide range in redshift $(0.3<z<2.2)$, and as yet there are insufficient statistics to determine whether this correlation length evolves with redshift (Croom \& Shanks 1996). At larger separations 
$\left(r>10 \mathrm{~h}^{-1} \mathrm{Mpc}\right)$ there are too few QSOs to identify any weak features which may be present in the correlation function (Shanks \& Boyle 1994). There have been some claims for QSO groups/clusters on $100 \mathrm{~h}^{-1} \mathrm{Mpc}$ scales (e.g. Clowes \& Campusano 1991), although the statistical significance of these associations is difficult to establish conclusively.

In using QSOs to study large-scale structure, an important observation is that radio-quiet QSOs exist in average galaxy clustering environments $\left(r_{0} \sim 5 \mathrm{~h}^{-1} \mathrm{Mpc}\right)$ at low-to-moderate redshifts (Smith et al. 1995 and references therein). This demonstrates that radio-quiet QSOs are likely to sample the galaxy distribution without significant bias, at least at $z<1$. In contrast, radio-loud QSOs inhabit richer environments (Ellingson et al. 1991) and exhibit much stronger clustering $\left(r_{0} \sim 12 \mathrm{~h}^{-1} \mathrm{Mpc}\right.$, see e.g. Loan et al. 1997). However, radio-loud QSOs comprise less than $5 \%$ of all QSOs with $B<21$ and are thus unrepresentative of the QSO population as a whole.

\section{Aims of the AAO 2dF QSO Redshift Survey}

With the advent of the 2-degree field (2dF), a 400-fibre multi-object fibre spectrograph at the Anglo-Australian Telescope (Taylor et al. 1994), it now possible to increase the size of existing QSO surveys by more than an order of magnitude. We have therefore embarked on a large spectroscopic survey of QSO candidates with the 2dF. The observational goal of this survey is to identify and obtain redshifts for $\sim 30000 B<21$ QSOs in two declination strips at the South Galactic Pole and in an equatorial region at the North Galactic cap. The QSO survey will probe the largest scales in the universe $\left(10 \mathrm{~h}^{-1} \mathrm{Mpc}<r<1000 \mathrm{~h}^{-1} \mathrm{Mpc}\right)$ over a wide range in redshift space $(0.3<z<2.9)$. The proposed survey will therefore increase the total number of QSOs known by a factor of $\sim 4$, and the number suitable for clustering studies by more than a factor of 10 .

The primary scientific aims of the survey are: a) to obtain the primordial fluctuation power spectrum out to COBE scales; $b$ ) to determine the rate of QSO clustering evolution in the non-linear and linear regimes, and hence obtain new limits on the value of $\Omega$ and $b$ (Croom \& Shanks 1996); c) to apply geometric methods to measure $\Lambda$ (Ballinger et al. 1996); d) to identify large statistical samples of unusual classes of QSOs (e.g. BALs) or absorption line systems (e.g. damped Ly $\alpha$ systems).

\section{Input Catalogue}

The $2 \mathrm{dF}$ survey covers a total area of $740 \mathrm{deg}^{2}$ comprising two $75^{\circ} \times 5^{\circ}$ strips on the sky (small areas surrounding bright stars have been excluded from the catalogue). QSO candidates were selected from APM measurements of 


$\begin{array}{llll}\text { UVX Input Catalogue for the 2dF_QSO_Redshift } & \text { Survey } \\ \text { Total area of survey } & : 740 \mathrm{deg}^{2} & \text { Number of candidates } & : 46077 \\ \text { Magnitude limits } & : 18.25 \leqq B_{\mathrm{J}} \leqq 21.0 & \text { Expected completeness } & : 90 \% \\ \text { Colour selection } & :\left(U-B_{J}\right) \leqq-0.36 & \text { Number of expected QSOs } & : 25000\end{array}$
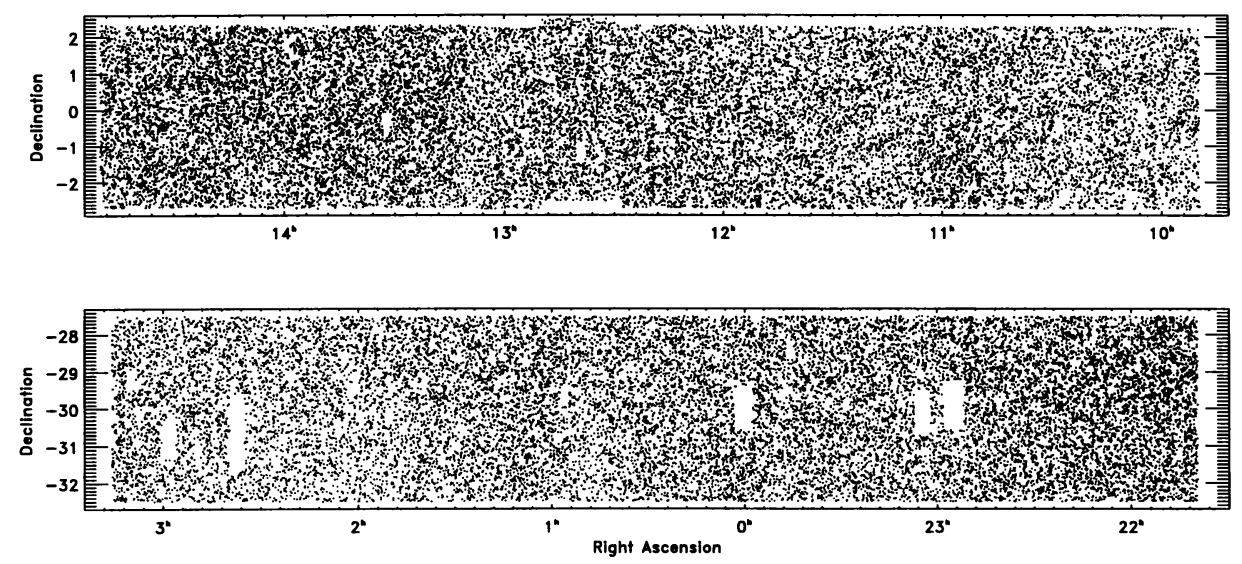

Figure 1. The UVX-selected objects for the QSO $2 \mathrm{dF}$ input catalogue. The RA and Dec of all UVX-selected objects are shown in the two $75^{\circ} \times 5^{\circ}$ strips of the catalogue. Increasing contamination by Galactic sub-dwarfs at lower Galactic latitudes gives an obvious gradient $(<50 \%)$ in number density along the survey strips. Completeness estimates are based on our success at recovering previously identified QSOs.

UK Schmidt $U, J$ and $R$ plates/films, on the basis of their $U-B / B-R$ colours. The vast majority $(>85 \%)$ of the candidates are selected solely on the basis of their $U-B$ colour (sensitive to QSOs with $z<2.2$ ), but the additional use of the $R$ magnitude minimises contamination from Galactic stars and extends the redshift range over which QSOs can be selected to $z \sim 2.9$. The initial UVX-selected catalogue is shown in Figure 1. In total over $150 \mathrm{U}, J$ and $R$ plates (comprising 30 UKST fields) were used to compile the input catalogue. Great care was taken to keep photometric variations at $<0.1 \mathrm{mag}$ level over the entire catalogue (Smith 1997).

\section{Survey Status}

The first 2dF observations for the QSO survey were made in January 1997, and since then almost 800 QSO candidates in the survey have been observed, comprising over 400 QSOs. Over 100 bright $(B<18)$ QSOs in the survey have been identified with the UKST FLAIR system and 20 QSOs in close pairs (separations less than 20 arcsec) have been discovered with 

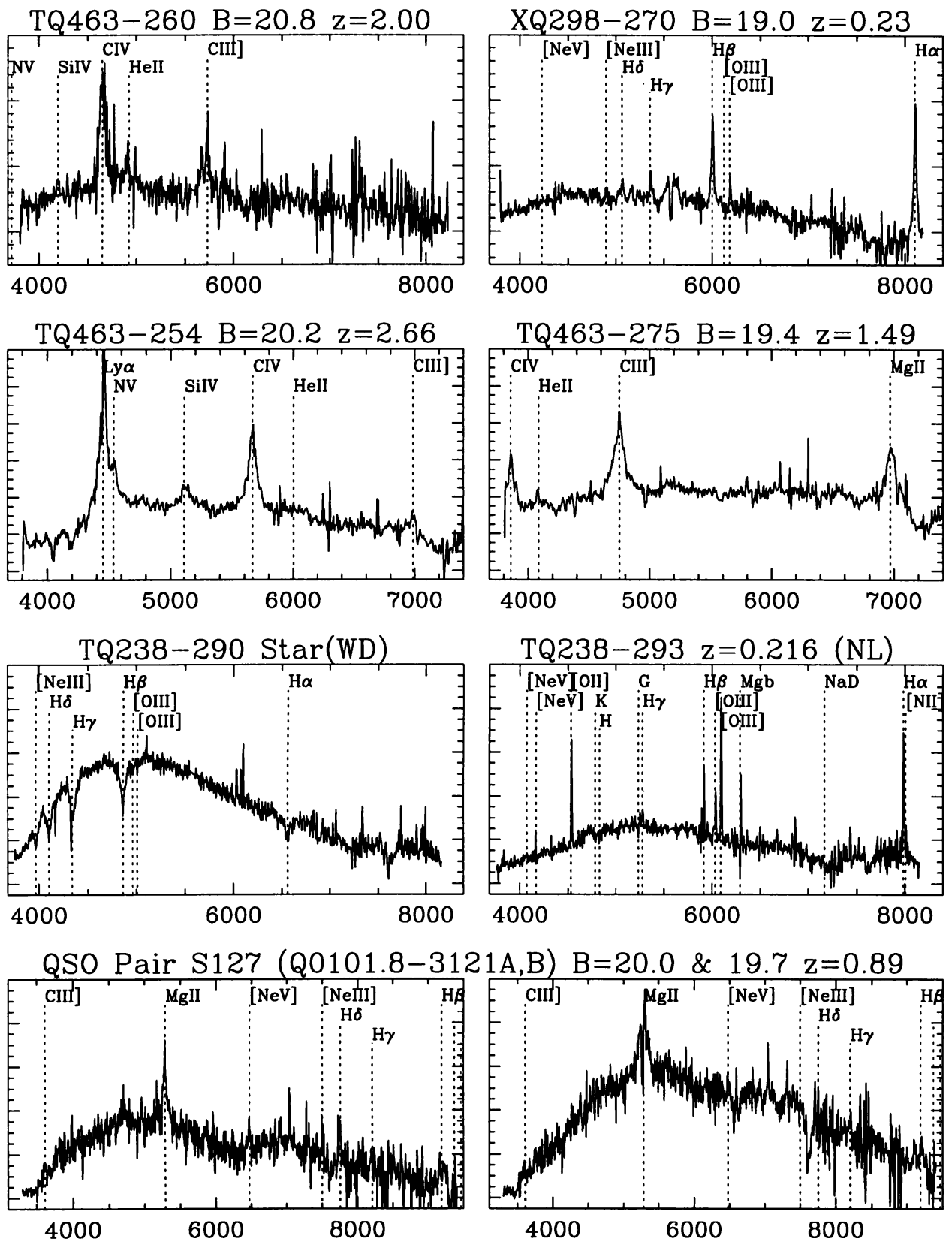

Observed Wavelength $(\AA)$

Figure 2. Representative spectra from the 2dF QSO redshift survey. The first six spectra were obtained with the $2 \mathrm{dF}$ and comprise 4 QSOs, a white dwarf and a narrow-emission-line galaxy. The latter two classes of object are some of the typical 'contaminants' of the UVX-selected sample. The bottom pair of objects are a close pair of QSOs (20 arcsec separation) observed with the MSSSO $2.3 \mathrm{~m}$. 


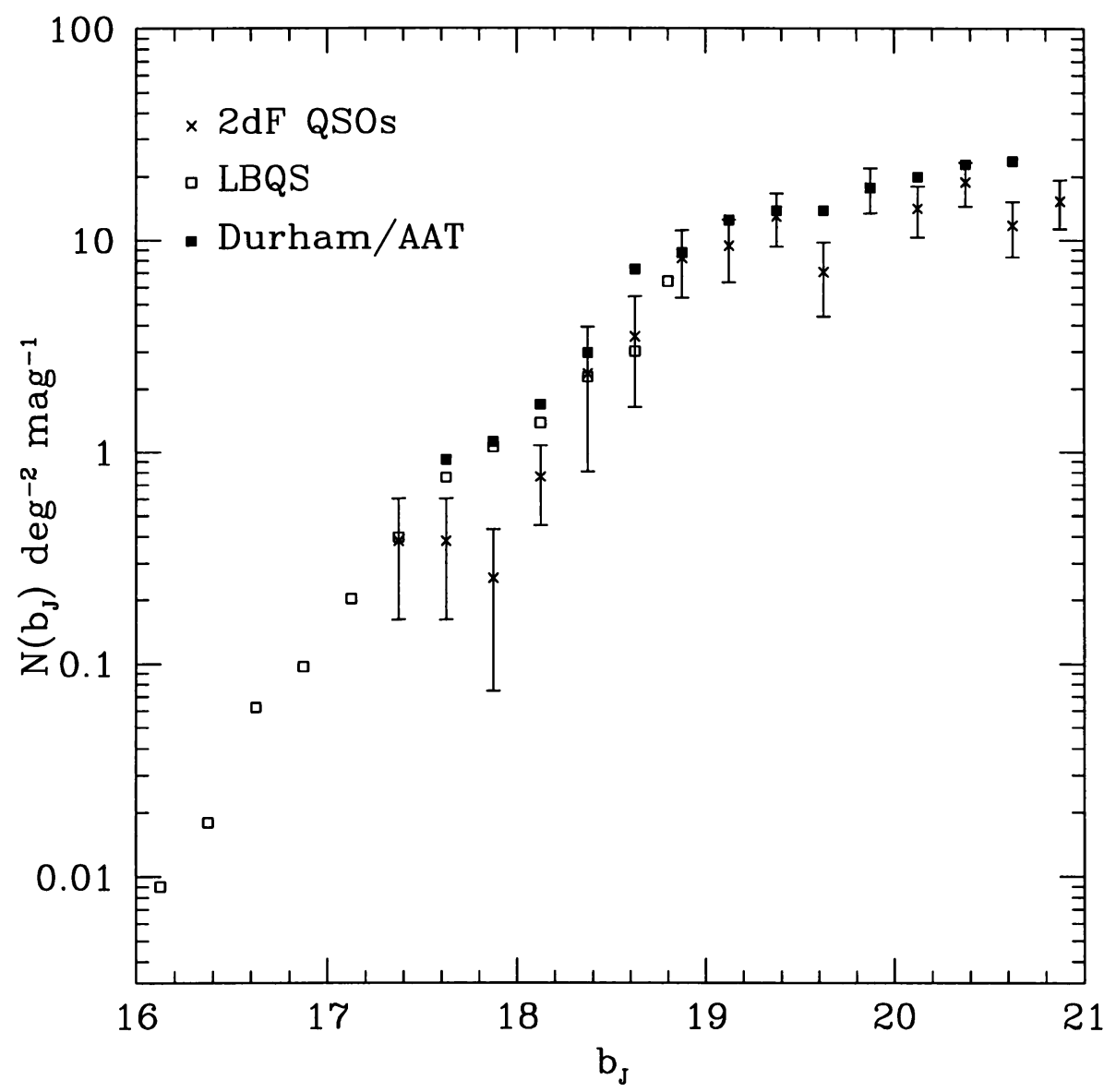

Figure 3. Number-magnitude relation for the first 114 QSOs in the 2dF redshift survey. The observed $n(m)$ relation for the Durham/AAT survey (Boyle et al. 1990) and LBQS (Hewett et al. 1995) is also shown.

the MSSSO 2.3m telescope. Representative spectra are shown in Figure 2.

The number-magnitude, $n(m)$, and number-redshift, $n(z)$ relations for the first 114 QSOs in the survey are shown in Figures 3 and 4 (see also Croom 1997). The $n(m)$ and $n(z)$ relations are consistent with those obtained from previous surveys.

During the current $2 \mathrm{dF}$ commissioning phase, the QSO survey progress has been relatively slow. However, as of September 1997, the full complement of 400 fibres and 2 spectrographs is now available, and, as the commissioning phase completes, the rate of progress will increase significantly. The aim is to complete the survey by the end of 1999 . 


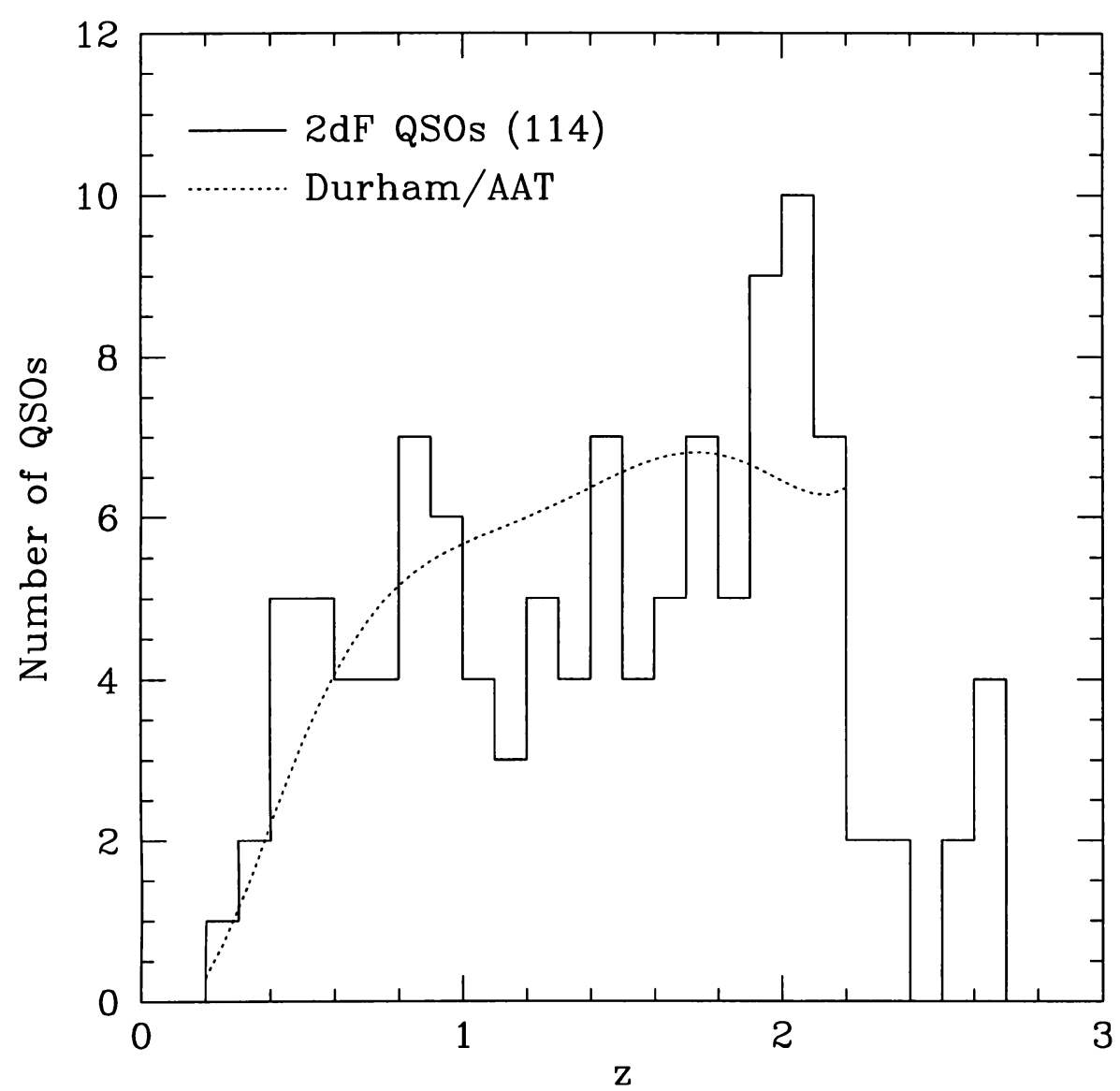

Figure 4. Number-redshift relation for the first 114 QSOs in the 2dF QSO redshift survey. The observed $n(z)$ relation for the Durham/AAT survey (Boyle et al. 1990) is also shown.

\section{References}

Ballinger W.E., Peacock J.A., Heavens A.F., 1996, MNRAS, 282, 877 Boyle B.J., Fong R., Shanks T., Peterson B.A., 1990, MNRAS, 243, 1 Clowes R.G., Campusano L.E., 1991, MNRAS, 249, 218

Crampton D., Cowley A.P., Hartwick F.D.A., 1989, ApJ, 345, 59

Croom S.M. 1997, PhD Thesis, University of Durham

Croom S.M., Shanks T., 1996, MNRAS, 281, 893

Ellingson E., Yee H.K.C., Green R.F., 1991, ApJ, 371, 49

Hamilton A.J.S., Matthews A., Kumar P., Lu E., 1991, ApJ, 374, 1

Hewett P.C., Foltz C.B., Chaffee F.H., 1995, AJ, 109, 1498

Loan A.J., Wall J.V., Lahav O., 1997, MNRAS, 286, 994

Shanks T., Boyle B.J., 1994, MNRAS, 271, 639 
Smith R.J., 1997, PhD Thesis, University of Cambridge

Smith R.J., Boyle B.J., Maddox S.M., 1995, MNRAS, 219, 537

Taylor K., 1994, in Wide Field Spectroscopy and the Distant Universe, eds Maddox S.J. et al., (World Scientific), p15

Veron-Cetty M.-P., Veron P., 1997, 'A catalogue of quasars and active nuclei', 7th edition, ESO Scientific Report 